\title{
Special Exceptions Process
}

National Cancer Institute

\section{Source}

National Cancer Institute. Special Exceptions Process. NCI Thesaurus. Code C20084.

A funding mechanism designed to ensure that new investigators share the same granting success rate as more established applicants. 